\title{
The distribution of the main risk factors in patients with intermediate-high risk pulmonary embolism
}

\author{
Alexandru Cristian Ion, Irina Dimitriu Cuciureanu, Catalina Liliana Andrei, \\ Crina Julieta Sinescu \\ "Carol Davila" University of Medicine and Pharmacy, Bucharest, Romania
}

\begin{abstract}
The pulmonary embolism (PE) represents a medical condition with increasing incidence. The various clinical forms of PE have different prognosis, related to the presence of markers of right ventricle (RV) dysfunction (biochemical or imagistic).

Material and method. In this study we included 82 consecutive patients with intermediate-high risk PE, assessing the main risk factors distribution. We divided the patients in two groups, study group - receiving thrombolytic therapy (ateplase (t-Pa)) associated to anticoagulation (unfractionated heparin (UFH)) - and control group - receiving anticoagulation alone -. The inclusion in study group was indicated by the high bleeding risk, in this group being included patients without contraindications for thrombolysis, patients without severe renal dysfunction and patients with body mass index (BMI) 18.5-29.9 kg/m2. We assessed the gender distribution in the two groups, the medium age distribution and the main PE risk factors in the two groups. Also, we assessed the effect of the both therapies on the hemodynamic instability rate on 7 day from admission (defined by systolic blood pressure (SBP) $<100 \mathrm{mmHg}$ or a SBP drop $>40 \mathrm{mmHg}$ from inclusion value). The statistical analysis was made using SPSS program, by comparing the association between risk factors and the two groups, by Chi-squared test, while the gender and age distribution was made using the Shapiro Wilk test for the evenly data distribution and Wilk test (as the data were unevenly distributed).

Results. We found no correlation between the risk factors and the study groups. There was no statistical significance regarding the gender distribution, but the medium age was higher in control group (61.82 y.o vs. 71.28 y.o, $p<0.001$. Regarding the hemodynamic instability rate the Chi-squared test proved a statistical significant higher incidence in the control group $(p=0.03)$.
\end{abstract}

Keywords: intermediate-high risk, pulmonary embolism, venous thromboembolism, risk factor, thrombolytic therapy, hemodynamic instability

\section{BACKGROUND}

PE represents a medical condition with increasing incidence, together with deep vein thrombosis (DVT) forming a continuum represented by venous thromboembolism (VTE). In large epidemiological studies the PE incidence is $39-115 / 100,000$ individuals, and for DVT 53-162/100,000 individuals $(1,2)$. Recent analysis show that the incidence of TED is eight times higher in patients over 80 y.o than the fifth life decade (2). Also, longitudinal studies reveal an increasing incidence rate of VTE in time (3). This fact, together with the hospital-associated costs and the indirect annual expenditures for VTE (an estimated value of 8.5 billion Euro in the European Union) (4), reveal the importance of $\mathrm{PE}$ and DVT in ageing Europe populations.

There is a large number of predisposing environmental and genetic factors for VTE. VTE is considered to be a consequence of the interaction between patient-related - usually permanent - risk factors and setting-related - usually temporary risk factors. The importance of this classification results from the impact of these factors on PE recurrence and anticoagulation period. According to Rogers et al. study (5) the risk factors were classified in strong (odds ratio $(\mathrm{OR})>10$ ), moderate (or 2-9) and weak $(\mathrm{OR}<2)$. In the strong risk factors class were included: fracture of lower limb, hospitalization for heart failure (HF) or respiratory in- 
sufficiency (RI) in the last 3 months, hip/knee replacement, major trauma, recent myocardial infarction - in the past 3 months -, spinal cord injury and history of VTE (6). In the moderate risk factor class were included known HF and IR without recent hospitalization, cancer (mainly in metastatic form), oral contraceptive and hormone replacement therapy, chemotherapy, severe infections, thrombophilia (7).

Major trauma, major surgery, lower limb fractures and hip/knee replacement, together with spinal cord injury were strong predisposing factors for VTE (7). Cancer is a known predisposing factor for PE, the VTE risk being different related to the cancer type: pancreatic tumors, gastric neoplasia, brain and lung tumors, together with hematological neoplasms having the highest VTE risk (8). Furthermore, neoplasia represents a risk factor for all cause mortality after VTE (9).

VTE can be considered as a part of cardiovascular continuum, as classic risk factors - smoking, obesity, hypercolesterolaemia, hypertension and diabetes mellitus $(10,11)$ - are also risk factors for atherosclerotic disease (12). Yet, it may be the cause of an indirect association through the complications of ischemic coronary disease and, regarding smoking, through neoplasia (13). The recent myocardial infarction and heart failure increase the PE risk (14).

Taking into account this considerations, the importance of studying the PE risk factors is essential related to their importance in PE assessment and risk evaluation. Meanwhile, the effect of the thrombolytic therapy on intermediate-high risk PE is promising, as the benefits can outweigh the risks.

\section{MATERIALS AND METHODS}

We included 82 consecutive patients with intermediate-high risk PE, as defined by the presence of both echocardiographic (right ventricle end-diastolic diameter (RVEDD) $>40 \mathrm{~mm}$, the RVEDD/ left ventricle end-diastolic diameter (LVEDD) ratio $>1$, tricuspid annulus pick systolic excursion (TAPSE) $<16 \mathrm{~mm}$, interventricular septum dyskinesia, the myocardial contraction velocity (MCV) of $\mathrm{RV}$ wall $<10 \mathrm{~cm} / \mathrm{s}$ ) and biochemical markers (brain natriuretic peptide $(\mathrm{BNP})>150 \mathrm{pg} / \mathrm{ml}$ ) of $\mathrm{RV}$ dysfunction in the absence of shock.

The inclusion criteria were: 1 . The first documented episode of PE. 2. Intermediate-high risk PE. 3. Age over 18 y.o. 4 . The ability to sign an informed consent. The exclusion criteria were: 1 .
Age over 85 y.o. 2. Previous PE. 3. Severe valvulopathies (severe aortic stenosis, severe mitral stenosis, severe mitral regurgitation) which may cause pulmonary hypertension. 4. High bleeding hematological disorders (end stage liver disease, haemophilia). 5. Severe anemic syndromes (serum hemoglobin $<8 \mathrm{~g} / \mathrm{dl}$ ). 6 . Severe psychiatric disorders, affecting the ability of informed consent. 7 . Diseases associated with life-expectancy lower than 1 year (end stage neoplasms). 8. Severe left ventricle dysfunction (left ventricle ejection fraction $(\mathrm{LVEF})<40 \%$ ).

The patients were assessed on admission regarding the pre-existing PE risk factors. The subjects were divided in two groups, study group receiving t-Pa and UFH - and control group - receiving only UFH -. In the study group were included patients with lower bleeding risk, without severe renal failure, with BMI 18.5-29.9 kg/m2. By this stratification patients with high bleeding risk were directed to anticoagulation alone, as both extreme BMI and severe renal failure are associated with bleeding risk. Meanwhile, we decided to exclude patients over 85 y.o, due to the high associated bleeding risk. The 7 day hemodynamic instability was assessed in the two groups, together with 7 day mortality rate.

The statistical analysis was made using SPSS program, by comparing the association between risk factors and the two groups, by Chi-squared test, while the gender and age distribution was made using the Shapiro Wilk test for the evenly data distribution and Wilk test (as the data were unevenly distributed). Regarding the hemodynamic instability rate the Chi-squared test proved a statistical significant higher incidence in the control group $(\mathrm{p}=0.03)$.

All the procedures and experiments of this study respect the ethical standards in the Helsinki Declaration of 1975 , as revised in 2008, as well as the national law. Informed consent was obtained from all the patients included in the study.

\section{RESULTS}

The first part of the statistical analysis included the comparative analysis of mean age between the two groups. The descriptive statistic showed a higher mean age in the control group compared to study group (61.85 vs. 71.28 y.o.) (Table 1).

In order to assess the significance of the mean age difference between the two groups, we first assessed the equality of data distribution through Shapiro-Wilk test. This test showed an unequal age distribution (Table 2). 
TABLE 1. Contingency table for age distribution in patients from study group and control group - personal data

\begin{tabular}{|l|r|r|}
\hline \multicolumn{3}{|c|}{ Age } \\
\hline & \multicolumn{1}{|c|}{ Study } & Control \\
\hline Valid & 39 & 43 \\
\hline Missing & 0 & 0 \\
\hline Mean & 61.85 & 71.28 \\
\hline Median & 64.00 & 74.00 \\
\hline Mode & 68.00 & 77.00 \\
\hline Std. Deviation & 13.09 & 10.29 \\
\hline IQR & 16.50 & 13.50 \\
\hline Minimum & 24.00 & 43.00 \\
\hline Maximum & 81.00 & 89.00 \\
\hline
\end{tabular}

TABLE 2. Shapiro-Wilk and Levene test for equality of variances regarding age distribution, showing an unequal distribution mean age - personal data

\begin{tabular}{|l|l|c|c|}
\hline \multicolumn{4}{|l|}{ Test of normality (Shapiro-Wilk) } \\
\hline & & $\mathbf{W}$ & $\mathbf{p}$ \\
\hline Age & Study & 0.93 & 0.02 \\
\hline & Control & 0.96 & 0.11 \\
\hline
\end{tabular}

\begin{tabular}{|l|c|c|c|}
\hline \multicolumn{4}{|c|}{ Test of equality of variances (Levene's) } \\
\hline & $\mathbf{F}$ & $\mathbf{d f}$ & $\mathbf{p}$ \\
\hline Age & 2.01 & 1 & 0.16 \\
\hline
\end{tabular}

The result of Shapiro-Wilk and Levene test show the unequal data distribution, therefore for the analysis of the statistical significance of mean age difference we use the Welch t-test (Table 3).

TABLE 3. Welch t-test for statistical significance of mean age difference between study group and control group

\begin{tabular}{|l|c|c|c|}
\hline \multicolumn{4}{|c|}{ Independent Samples T-Test } \\
\hline & $\mathbf{t}$ & $\mathbf{d f}$ & $\mathbf{p}$ \\
\hline Age & -3.60 & 72.05 & $<.001$ \\
\hline
\end{tabular}

Note. Welch's t-test

The mean age in the control group was statistical significant higher than study group, this difference being explained by inclusion criteria, as patients with higher bleeding risk were included in control group, as both extreme BMI and severe renal dysfunction are associated with older age.

Regarding the gender distribution, the statistical analysis was made through Chi-squared test, showing no statistical significant difference between the two groups regarding gender distribution.

TABLE 5. Chi-squared test for gender distribution in study and control group - personal data

\begin{tabular}{|l|r|r|c|}
\hline \multicolumn{4}{|c|}{ Chi-Squared tests } \\
\hline & Value & df & p \\
\hline $\mathrm{X}^{2}$ & 0.15 & 1 & 0.70 \\
\hline $\mathrm{N}$ & 82 & & \\
\hline
\end{tabular}

There was no statistical significant difference regarding sex distribution in the two groups $(\mathrm{p}=0.7)$.

Next, we assessed the incidence of risk factors in the two groups and the possible association between these factors and any of the two groups. The main risk factors identified were HF or RI (51.28\% study group vs. $60.46 \%$ in control group), neoplasia $(35.9 \%$ study group vs. $41.86 \%$ in control group), lower limb fractures (23.07\% study group vs. $27.9 \%$ in control group) and hip $/$ knee replacements $(23.07 \%$ study group vs. $25.58 \%$ control group).

Regarding the HF or RI incidence the higher rate in control group is justified by the higher mean age in this group compared to study group, as both $\mathrm{HF}$ and RI are related to increasing age. The statistical significance of this difference was assessed through Chi-squared test (Table 6).

The Chi-squared test showed no statistical difference between the two groups regarding the HF or RI incidence, even if the incidence was higher in the control group $(51.28 \%$ vs. $60.46 \%, \mathrm{p}=0.4)$. This finding sustains the right patient selection as both HF and RI can be associated with poor prognosis in patients.

TABLE 4. Frequency distribution for gender in study and control group - personal data

\begin{tabular}{|c|l|r|r|r|r|}
\hline \multicolumn{1}{|c|}{ Frequencies for sex } \\
\hline Group & Sex & Frequency & Percent & $\begin{array}{c}\text { Valid } \\
\text { Percent }\end{array}$ & $\begin{array}{c}\text { Cumulative } \\
\text { Percent }\end{array}$ \\
\hline Study & F & 21 & 53.85 & 53.85 & 53.85 \\
\hline & M & 18 & 46.15 & 46.15 & 100.00 \\
\hline & Missing & 0 & 0.00 & & \\
\hline & Total & 39 & 100.00 & & \\
\hline Control & F & 25 & 58.14 & 58.14 & 58.14 \\
\hline & M & 18 & 41.86 & 41.86 & 100.00 \\
\hline & Missing & 0 & 0.00 & & \\
\hline & Total & 43 & 100.00 & & \\
\hline
\end{tabular}


TABLE 6. Contingency table for HF and RI incidence in the two groups. Chi-squared test for statistical significance of the difference between the two groups - personal data

\begin{tabular}{|c|c|c|c|}
\hline \multicolumn{4}{|c|}{ Contingency tables } \\
\hline & \multicolumn{2}{|c|}{ RI, HF } & \multirow[b]{2}{*}{ Total } \\
\hline Group & Yes & No & \\
\hline Study & 20 & 19 & 39 \\
\hline Control & 26 & 17 & 43 \\
\hline Total & 46 & 36 & 82 \\
\hline \multicolumn{4}{|c|}{ Chi-Squared tests } \\
\hline & Value & df & $p$ \\
\hline$x^{2}$ & 0.70 & 1 & 0.40 \\
\hline $\mathrm{N}$ & 82 & & \\
\hline
\end{tabular}

The analysis of neoplasia distribution in the two groups showed also a higher incidence in the control group (Table 7), this difference being also explained by the higher mean age in the control group, because of the inclusion criteria.

Using the Chi-squared test we assessed the statistical significance of the difference between the two groups regarding the neoplasia incidence (35.9\% vs. $41.86 \%, \mathrm{p}=0.58)$ (Table 7). The Chisquared test showed no statistical difference between these two groups, even if the incidence was higher in the control group. The main neoplasia was brain tumors (10), followed by gastric neoplasia (8).

TABLE 7. Chi-squared test regarding the neoplasia distribution in the two groups, with no statistical significant result

\begin{tabular}{|c|c|c|c|c|}
\hline \multirow[b]{2}{*}{ Group } & \multicolumn{3}{|c|}{ RF neoplasia } & \multirow[b]{2}{*}{ Total } \\
\hline & \multicolumn{2}{|c|}{$\mathrm{Da}$} & $\mathrm{Nu}$ & \\
\hline Study & & 14 & 25 & 39 \\
\hline Control & & 18 & 25 & 43 \\
\hline Total & & 32 & 50 & 82 \\
\hline \multicolumn{5}{|c|}{ Chi-Squared Tests } \\
\hline \multicolumn{2}{|c|}{ Value } & df & $p$ & \\
\hline$x^{2}$ & 0.31 & 1 & 0.58 & \\
\hline $\mathrm{N}$ & 82 & & & \\
\hline
\end{tabular}

Regarding the hip/knee replacement, the incidence of this risk factor was higher in the control group, caused by the increasing incidence with age. There was no statistical significance between the two groups regarding the hip/knee replacement incidence $(23.07 \%$ vs. $25.58 \%, p=0.79)$.

The lower limb fractures were more frequent in the control group. Using the Chi-squared test we found no statistical difference regarding the lower limb fracture in the two groups $(23.07 \%$ vs. $27.9 \%$, $\mathrm{p}=0.62$ ).

Other risk factors had a much lower incidence, the statistical analysis was not assessed for this factors (thrombophilia 1 patient in study group, 2 patients in control group; severe infection 1 patient in each group; oral contraceptive therapy 1 patient in each group). The last part of the statistical analysis took into account the 7 day hemodynamical instability rate, as a prognostic marker of PE patients. We did not assess the early mortality due to the relative low number of patients, and the short following period. The assessment of the 30 day mortality can be biased by the type of oral anticoagulant (novel anticoagulant (NoAc) vs. Vitamin $\mathrm{K}$ antagonists (VKA)) and by the patient compliance to the anticoagulation therapy. We assessed the 7 day hemodynamic instability rate through the Chi squared test, the control group being associated with a higher incidence of hemodynamic instability $(10.26 \%$ study group vs. $30.23 \%$ control group, $\mathrm{p}=0.03)$, this difference being statistical significant (Table 8).

TABLE 8. The contingency table and Chi-squared test for hemodynamic instability in the two groups, showing a statistical significant difference between the two groups

\begin{tabular}{|c|c|c|c|c|}
\hline \multicolumn{5}{|c|}{ Contingency Tables } \\
\hline \multirow[b]{2}{*}{ Grup } & \multicolumn{2}{|c|}{ Hemodynamic instability- } & \multirow{2}{*}{\multicolumn{2}{|c|}{ Total }} \\
\hline & 0 & 1 & & \\
\hline Study & 35 & 4 & \multicolumn{2}{|r|}{39} \\
\hline Control & 30 & 13 & & 43 \\
\hline Total & 65 & 17 & & 82 \\
\hline \multicolumn{5}{|c|}{ Chi-Squared Tests } \\
\hline & & Value & df & $\mathbf{p}$ \\
\hline \multicolumn{2}{|c|}{$x^{2}$} & 4.97 & & 0.03 \\
\hline \multicolumn{2}{|c|}{$\mathrm{N}$} & 82 & 1 & \\
\hline
\end{tabular}

To conclude, we identified no statistical significant difference regarding the main risk factor distribution in the two groups, even if the incidence of this factors was higher in the control group. This result confirms the proper patient selection, as risk factors can also influence patient's prognosis. Meanwhile, the hemodynamic instability was lower in the study group, suggesting a positive effect of thrombolytic therapy in these patients.

\section{DISCUSSIONS}

The risk factors in PE are a very important phase in evaluating patients. These risk factors can influence the patient prognosis and the patient risk for recurrence. In our study we found the main risk factors as being HF and RI, neoplasia, hip/knee replacement and lower limb fracture. In an analysis by Chew and al. (17) the incidence of PE in a group of 235,149 cancer cases was $(1.6 \%)$ within 2 years, $463(12 \%)$ at the time cancer was diagnosed and 
$3,312(88 \%)$ subsequently. In risk-adjusted models, metastatic disease at the time of diagnosis was the strongest predictor of thromboembolism. Adjusting for age, race, and stage, diagnosis of thromboembolism was a significant predictor of decreased survival during the first year for all cancer types (hazard ratios, 1.6-4.2; $\mathrm{p}<0.01$ ).

This result in concordant with our study, as one of the most frequent risk factors was neoplasia, without any difference regarding the study and control group, as the neoplasia incidence was comparable, without any statistical significance.

Regarding HF, in a nationwide study by Sørensen et al. (14) conducted during 17 years, myocardial infarction and heart failure in the preceding 3 months conferred high risks of apparently isolated pulmonary embolism (OR, 43.5 [95\% confidence interval (CI), 39.6-47.8] and 32.4 [95\% CI, 29.8-35.2], respectively), whereas the risks of combined pulmonary embolism and deep venous thrombosis (19.7 [95\% CI, 16.0-24.2] and 22.1 [95\% CI, 18.7-26.0], respectively) and deep venous thrombosis alone (9.6 [95\% CI, 8.6-10.7] and 12.7 [95\% CI, 11.6-13.9], respectively) were lower. Left-sided valvular disease was associated with an odds ratio of 13.5 (95\% CI, 11.3-16.1), whereas the odds ratio was 74.6 (95\% CI, 28.4195.8) for right-sided valvular disease. This result is also concordant to our findings, as HF and RI was the main risk factor for PE in our study, because of the same pathophysiological pathways and even similar risk factors.

Regarding the impact of thrombolysis on hemodynamical instability in patients with intermediate-high risk PE, we will assess the present data regarding the effect of this therapy. A large 15 studies meta-analysis including 2,057 patients evaluated the impact of thrombolytic therapy compared to UFH alone on all-cause mortality (15). The thrombolytic therapy was associated with a significant decrease of all-cause mortality (OR: $0.59 ; 95 \%$ confidence interval (CI): 0.36-0.96). This effect was not statistical significant after excluding the studies including high risk PE (OR 0.64 ; 95\% CI: $0.35-1.17)$. The thrombolytic therapy was associated with a statistical significant decrease of the composit end-point mortality - hemodynamic instability (OR: $0.34 ; 95 \%$ CI: $0.22-0.53$ ) but also of the PE related mortality rate (OR: 0.50; 95\% CI : 0.27-0.94). The major bleeding rate was statisticaly significant higher in the thrombolysed patients (OR: 2.91, 95\% CI: $1.95-$ 4.36) (15). In the PEITHO study the thrombolytic therapy was associated with a reduction of hemod- ynamic instability risk, but with an increase of the major bleeding rate. The 30-day mortality rate showed no statistical significant difference between the thwo groups (16).

Compared to the results from Marti et al. meta-analysis (15), including a high number of patients, the hemodynamic instability rate was lower in thrombolysed patients from our study, compared to a significant decrease of the composit end-point mortality- hemodynamic instability in the meta-analysis assessed (OR: 0.34; 95\% CI: 0.22-0.53). This difference is explained by the right patient selection, with a very good risk/benefit profile, which led to a decrease of hemodynamic instability rate in the thrombolysed patients. In the PEITHO study the thrombolytic therapy was associated with a reduction of hemodynamic instability risk, but with an increase of the major bleeding rate. The 30-day mortality rate showed no statistical significant difference between the two groups (16). In comparison, in our study, the 30 day mortality was not assessed as it can be biased by the type of oral anticoagulation the patients follow in the subacute phase and the compliance to this therapy.

As strong points of our study, the revealing of the main risk factors in patients with intermediary-high risk PE is a very important clinical feature, as risk factors have a very important role in diagnosing PE. Meanwhile, the evaluation of the 7 day hemodynamic instability reveals the effect of thrombolysis compared to anticoagulation alone, without any bias resulting from oral anticoagulation.

As weak points we can include the low number of patients, because this is a monocentric study, and the lack of data regarding 30 day mortality (as it may be influenced by oral anticoagulation and the patient compliance to this therapy). The 7 day mortality was not assessed as the short treatment period could not lead to any statistical significant result.

\section{CONCLUSIONS}

To conclude, the evaluation of the risk factors for intermediate-high risk PE is a very important step in assessing patients with this pathology. We found no difference between the two groups thrombolysed vs. anticoagulation alone-, this fact suggesting that patient initial prognosis was similar.

Meanwhile, the positive effect of thrombolytic therapy on hemodynamic instability sustains the benefit of further investigations on this field, the permanent assessment of risk/benefit ratio being the key to the optimal clinic effect. 


\section{REFERENCES}

1. Wendelboe AM, Raskob GE. Global Burden of Thrombosis: Epidemiologic Aspects. Circ Res. 2016 Apr 29;118(9):1340-7.

2. Keller K, Hobohm L, Ebner M, Kresoja KP, Münzel T, Konstantinides $\mathrm{SV}$, Lankeit M. Trends in thrombolytic treatment and outcomes of acute pulmonary embolism in Germany. Eur Heart J. 2020 Jan 21;41(4):522-529.

3. de Miguel-Díez J, Jiménez-García R, Jiménez D, Monreal M, et al. Trends in hospital admissions for pulmonary embolism in Spain from 2002 to 2011. Eur Respir J. 2014 Oct;44(4):942-50.

4. Barco S, Woersching AL, Spyropoulos AC, Piovella F, Mahan CE. European Union-28: An annualised cost-of-illness model for venous thromboembolism. Thromb Haemost. 2016 Apr;115(4):800-8.

5. Rogers MA, Levine DA, Blumberg N, Flanders SA, Chopra V, Langa $\mathrm{KM}$. Triggers of hospitalization for venous thromboembolism. Circulation. 2012 May 1;125(17):2092-9.

6. Konstantinides SV, Meyer G, Becattini C, Bueno H, Geersing GJ, Harjola VP, et al.; ESC Scientific Document Group. 2019 ESC Guidelines for the diagnosis and management of acute pulmonary embolism developed in collaboration with the European Respiratory Society (ERS). Eur Heart J. 2020 Jan 21;41(4):543-603.

7. Anderson FA Jr, Spencer FA. Risk factors for venous thromboembolism. Circulation. 2003 Jun 17;107(23 Suppl 1):19-16.

8. Ku GH, White RH, Chew HK, Harvey DJ, Zhou H, Wun T. Venous thromboembolism in patients with acute leukemia: incidence, risk factors, and effect on survival. Blood. 2009 Apr 23;113(17):3911-7.

9. Gussoni G, Frasson S, La Regina M, Di Micco P, Monreal M; RIETE Investigators. Three-month mortality rate and clinical predictors in patients with venous thromboembolism and cancer. Findings from the RIETE registry. Thromb Res. 2013 Jan;131(1):24-30.

10. Piazza G, Goldhaber SZ. Venous thromboembolism and atherothrombosis: an integrated approach. Circulation. 2010 May 18;121(19):2146-50.

\section{Conflict of interest: none declared Financial support: none declared}

11. Ageno W, Becattini C, Brighton T, Selby R, Kamphuisen PW. Cardiovascular risk factors and venous thromboembolism: a meta-analysis. Circulation. 2008 Jan 1;117(1):93-102.

12. Gresele P, Momi S, Migliacci R. Endothelium, venous thromboembolism and ischaemic cardiovascular events. Thromb Haemost. 2010 Jan;103(1):56-61.

13. Enga KF, Braekkan SK, Hansen-Krone IJ, le Cessie S, Rosendaal FR, Hansen JB. Cigarette smoking and the risk of venous thromboembolism: the Tromsø Study. J Thromb Haemost. 2012 Oct;10(10):2068-74.

14. Sørensen HT, Horvath-Puho E, Lash TL, Christiansen CF, Pesavento $R$, Pedersen L, Baron JA, Prandoni P. Heart disease may be a risk factor for pulmonary embolism without peripheral deep venous thrombosis. Circulation. 2011 Sep 27;124(13):1435-41.

15. Marti C, John G, Konstantinides S, Combescure C, Sanchez O, Lankeit M, Meyer G, Perrier A. Systemic thrombolytic therapy for acute pulmonary embolism: a systematic review and meta-analysis. Eur Heart J. 2015 Mar 7;36(10):605-14.

16. Meyer G, Vicaut E, Danays T, Agnelli G, Becattini C, BeyerWestendorf J, Bluhmki E, et al.; PEITHO Investigators. Fibrinolysis for patients with intermediate-risk pulmonary embolism. N Engl J Med. 2014 Apr 10;370(15):1402-11.

17. Chew HK, Wun T, Harvey D, Zhou H, White RH. Incidence of venous thromboembolism and its effect on survival among patients with common cancers. Arch Intern Med. 2006 Feb 27;166(4):458-64. 